\title{
Relations Between Hopelessness, Depressive Symptoms and Suicidality: Mediation by Reasons for Living
}

\author{
Courtney L. Bagge, ${ }^{1}$ Dorian A. Lamis, ${ }^{2}$ Michael Nadorff, ${ }^{3}$ and Augustine Osman ${ }^{4}$ \\ ${ }^{1}$ University of Mississippi Medical Center \\ ${ }^{2}$ Emory University School of Medicine/Grady Health System \\ ${ }^{3}$ Mississippi State University \\ ${ }^{4}$ University of Texas at San Antonio
}

Objective: The present study examined whether reasons for living (RFL) would partially account for the associations between traditional risk factors (depressive symptoms, hopelessness) and suicidal ideation and attempts. Method: Data were collected from 1,075 undergraduate college students who completed a battery of online assessments. Results: Results from a series of simultaneous mediational models indicated that the relations between risk factors and current suicidal ideation were partially mediated by total RFL (and Coping Beliefs and Self-Evaluation subscales). Further, total RFL (and the Coping Beliefs subscale) fully mediated the relation between hopelessness and past-year suicide attempt, and partially mediated the depressive symptoms-suicide attempt relation. Conclusions: This study demonstrates the importance of assessing for the presence of these suicide risk and protective factors. Implications for the improved identification and treatment of young adults at risk for suicide are discussed. (c) 2013 Wiley Periodicals, Inc. J. Clin. Psychol. 00:1-14, 2013.

Keywords: suicidal ideation; suicide attempt; reasons for living; hopelessness; college students

Suicide is a major public health concern in the United States and is the third leading cause of death among young adults (aged 18-24 years; Xu, Kochanek, Murphy, \& Tejada-Vera, 2010), accounting for approximately 3,500 suicides each year (Center of Disease Control and Prevention [CDC], 2010). Rates of suicidal ideation and attempts are also of interest within this age group, particularly among those attending college. Specifically, approximately $20 \%$ of college students report seriously considering suicide, whereas over $7 \%$ report attempting suicide in their lifetime (American College Health Association, 2011). Moreover, in a recent study of over 15,000 undergraduates at 70 colleges and universities, $6 \%$ reported having seriously considered committing suicide in the past 12 months (Drum, Brownson, Burton Denmark, \& Smith, 2009) and $1.7 \%$ reported making a suicide attempt in the past year (Barrios, Everett, Simon, \& Brener, 2000). Given the importance of preventing suicidal ideation and behavior (i.e., suicide attempts and completions), a large body of research has focused on identifying reliable risk markers, or predictors, of suicidality in this age group. Both depression and hopelessness about the future, in particular, have been identified as robust psychological risk factors for suicidal ideation and behavior (see Haney et al., 2012 for review; National Institute of Mental Health [NIMH 2011]), and these factors are frequently targeted in prevention and treatment programs of suicidality (e.g., Drum \& Burton Denmark, 2011; Jobes, 2006; Wenzel, Brown, \& Beck, 2008). However, from a prevention and treatment perspective, it is imperative to supplement the identification of risk factors with the examination of potential mechanisms underlying associations between known risk factors (e.g., depression and hopelessness) and suicidal ideation and behavior.

A number of theoretical models of suicidal behavior (Joiner, 2005; Schotte, Cools, \& Payvar, 1990; Williams, Barnhofer, Crane, \& Beck, 2005) have posited that cognitive processes are important mediating variables in understanding suicidal risk. One cognitive factor, the importance assigned to reasons for living (RFL; Linehan, Goodstein, Nielson, \& Chiles, 1983)

Please address correspondence to: Courtney L. Bagge, University of Mississippi Medical Center, Department of Psychiatry and Human Behavior, Jackson, MS, 39216. E-mail: cbagge@umc.edu 
holds particular promise in furthering our knowledge of how beliefs of those who are at risk for suicidal behavior influence the engagement of suicidal actions. RFL are defined as reasons for not committing suicide despite suicidal thoughts or considerations (Linehan et al., 1983).

Thus, suicide-specific (low life affirming) cognitive/evaluative processes may be a key component in determining how depressive symptoms and hopelessness relate to suicidal thoughts and behavior. As such, individuals who are hopeless about the future and/or have depressive symptoms may have difficulty identifying, enumerating, or connecting to RFL and, as a result, may seriously consider suicide and/or increase the self-reported likelihood of making a future suicide attempt (e.g., Gutierrez et al., 2002). Research consistently demonstrates that increases in depressive symptoms and hopelessness are not only related to increases in suicidal ideation and presence of a suicide attempt history but also are associated with low RFL across clinical (e.g., Dean \& Range, 1999; Malone et al., 2000) and community populations (e.g., Dyck, 1991; Ellis \& Lamis, 2007; Gutierrez et al., 2002; Rich \& Bonner, 1987). Furthermore, RFL protect against (i.e., are negatively correlated with) suicidal thoughts and behavior concurrently (Chapman, Specht, \& Cellucci, 2005; Dean \& Range, 1999; Gutierrez et al., 2002; Dyck, 1991; Linehan et al., 1983; Mann, Waternaux, Haas, \& Malone, 1999; Malone et al., 2000; Rich \& Bonner, 1987) and are prospective predictors of suicidal behavior (Galfalvy et al., 2006).

One key preliminary investigation (Gutierrez et al., 2002) provides initial support for statistical mediation and demonstrated that increases in hopelessness was associated with increases in selfreported suicide likelihood, and that this relation was partially mediated by low RFL in a young adult sample. Similarly, Wang, Lightsey, Pietruszka, Uruk, and Wells (2007) found RFL to be a significant mediator in the relations between stress, coping, and suicidal behavior in a sample of undergraduate college students. It is important to highlight that hopelessness and RFL, although negatively correlated cognitive processes, show distinct nomological nets in relation to suicidality, thus indicating their distinctiveness (Dyck, 1991). However, to our knowledge no investigation to date has formally examined whether RFL simultaneously mediate the relations between key validated risk factors (depressive symptoms and hopelessness) and both suicidal thoughts and attempts. Therefore, the first goal of the current study was to use simultaneous tests of mediation to determine whether RFL may partially account for both of these associations in a large sample of young adults.

There is evidence to suggest that various RFL categories may have differential importance for suicidal ideation and behavior. Several categories of RFL have been identified specifically for young adult populations (Gutierrez, Osman, Kopper, Barrios, \& Bagge, 2000) that contain reasons for not killing oneself pertaining to: (a) family relations (i.e., one's relationship with his/her family), (b) peer relations (i.e., one's relationships with peers, (c) survival and coping beliefs (SCB; i.e., one's ability to cope and adjust to life's problems); (d) future expectations (i.e., one's outlook toward the future), and (e) self-evaluation (i.e., one's assessment of perceived positive self-appraisal). Previous research suggests that positive SCB, future expectations, and family relations are uniquely associated with less suicidal ideation (Dogra, Basu, \& Das, 2011) and that only positive SCB are uniquely associated with absence of a suicide attempt history (Chapman et al., 2005) when accounting for other RFL subscales. Thus, studies examining specific categories of RFL find that one category, SCB, has unique associations with both suicidal thoughts and behaviors.

Such research shows that individuals with suicidal thoughts or behaviors have less positive beliefs/expectations about their ability to survive and cope with life. Interestingly, SCB was the only RFL subscale rated lower among hospitalized single-car accident drivers, compared with an accident-free control group. Although cases and controls did not differ on self-reported suicidal risk, single-car accident drivers reported more impactful negative life events and reported being tired of living more often than controls presenting to the Emergency Department in Rome, Italy, suggesting that the accident may have been contemplated as a solution to their problems (Pompili, Girardi, Tatarelli, \& Taterelli, 2006). Although these results are from a study conducted in Italy, and caution must be exercised when generalizing the findings to U.S. samples, these findings are consistent with those findings from a U.S. psychiatric sample, which demonstrated that patients who viewed suicide as a solution to their problems reported lower SCB RFL (Linehan et al., 1983). 
Not only are SCB negatively associated with suicidal thoughts and behavior, but suicide risk factors (depressive symptoms and hopelessness) are also negatively associated with SCB (Ellis \& Lamis, 2007; Strosahl, Chiles, \& Linehan, 1992). Consistent with our mediation hypothesis, among a sample of recently hospitalized suicide attempters (Strosahl et al., 1992), SCB, but not hopelessness and depressive symptoms, were uniquely associated with suicide intent when all three constructs were included in the final step of the regression model. Conversely, within a sample of inmates, hopelessness, but not SCB, was uniquely associated with a lifetime history of suicide attempt (Chapman et al., 2005). Given the preliminary and conflicting nature of these data, the second goal of the current study was to determine whether SCB RFL, in particular, can partially explain the association between risk factors and suicidal ideation and suicidal behavior using simultaneous tests of mediation among a sample of young adults.

If RFL can at least partially explain the connection between key risk factors (hopelessness and depressive symptoms) and suicidal thoughts and behaviors, then they may be an ideal target for intervention. Because a clinician can aid patients in identifying and bolstering current RFL, directly targeting an increase in this protective factor may help to weaken the link between depressive symptoms, hopelessness, and suicide. As such, the current study has significant clinical relevance, as it has the potential to help clarify the relations between depressive symptoms, hopelessness, and suicidal ideation and behavior, and it may lead to the development of improved treatment strategies for reducing suicidality among depressed or hopeless individuals. In the current study, we aim to build upon previous research by examining in a large sample of young adults (a) whether total RFL mediates the relation between risk factors (hopelessness and depressive symptoms) and both suicidal ideation and attempt, and (b) whether specific categories of RFL uniquely mediate the relation between risk factors and suicide ideation and attempt. Based on the literature to date, we hypothesize that RFL, specifically SCB, would partially mediate the relations between both depressive symptoms and hopelessness, and suicidal ideation and suicide attempt.

\section{Method}

\section{Participants}

Participants comprised 1,075 undergraduate psychology students at a large southeastern university. Demographic characteristics of the sample were as follows: $69.3 \%$ female, $78 \%$ European American, 13\% African American, 4\% Asian American, 1\% Hispanic, and 4\% other. The average age was 19.28 years (median $=19.0$, standard deviation $[S D]=1.23$ ) and the sample comprised college freshmen $(47.5 \%)$, sophomores $(28.1 \%)$, juniors $(14.5 \%)$, and seniors $(9.9 \%)$.

\section{Procedure}

Data collection was conducted through an online survey over the course of two semesters, with approximately equal numbers of participants completing the study during the fall and spring. Participants voluntarily chose to complete the survey outside of class time in return for extra credit in their psychology course. Students were told of the study in regularly scheduled classes and through a posting on the online participant pool site. Participants completed a demographic survey and the study measures, which were presented in a randomized order. Prior to data collection, electronic informed consent was obtained from participants. The university's institutional review board approved the study in advance of data collection, and ethical procedures were followed throughout the study.

\section{Measures}

Beck Hopelessness Scale (BHS). The BHS (Beck, Weissman, Lester, \& Trexler, 1974) is a 20-item self-report measure of hopelessness or negative attitudes about future-related events. Example items on the BHS include "I have enough time to accomplish the things I want to do" and "I look forward to the future with hope and enthusiasm." Each item is rated as either 
true or false and scores are summed to derive a total scale score. High scores are indicative of stronger negative attitudes about the future. Studies with the BHS have reported acceptable estimates of internal consistency and concurrent validity for the total BHS score (Bisconer \& Gross, 2007; Brown, Henriques, Sosdjan, \& Beck, 2004). Moreover, there is strong evidence for convergent validity as demonstrated by high correlations between the BHS and similar constructs in university students (Steed, 2001).

Beck Depression Inventory - II (BDI-II). The BDI-II (Beck, Steer, \& Brown, 1996) is a widely used 21-item self-report measure of the severity of depressive symptoms. The items (groups of specific statements) are scored from 0 to 3 to assess the level of symptom severity over the course of the past 2 weeks. Each item measures a distinct depressive symptom (e.g., sad mood) through a series of four statements that reflect greater severity as they progress (e.g., "I do not feel sad," "I feel sad," "I am sad all the time," or "I am so sad or unhappy that I can't stand it"). Responses on the items are summed to derive a total scale score, with higher scores suggestive of higher depressive symptom severity. Good estimates of internal consistency and concurrent validity have been demonstrated in clinical and nonclinical samples (Bisconer \& Gross, 2007; Naragon-Gainey, Watson, \& Markon, 2009). For example, Osman, Kopper, Barrios, Gutierrez, and Bagge (2004) found that scores on BDI-II were correlated with measures of suicide risk and other measures of depression. Consistent with previous studies (e.g., Keilp et al., 2012; Pukay-Martin et al., 2012), in order to avoid criterion contamination, item 9 "Suicidal thoughts and wishes" was not included in the summed total score. ${ }^{1}$

RFL Inventory for Young Adults (RFL-YA). The RFL-YA (Gutierrez et al., 2000) comprises 32 items that are constructed to assess reasons young adults give for not engaging in suicide-related behaviors (i.e., reasons for living). Each item is rated on a 6-point scale ranging from 1 (not at all important) to 6 (extremely important). Responses on the items are averaged to obtain a total scale score and five subscale scores; higher scores are indicative of stronger reasons for living. Factor analyses have supported the 5-factor scales, which include family relations (e.g., "I have a close relationship with my family"), peer relations (e.g., "my friends stand by me whenever I have a problem"), SCB (e.g., "when faced with a problem I work hard to avoid similar problem situations"), future expectations (e.g., "I have many plans that I am looking forward to carrying out in the future"), and self-evaluation (e.g., "I am happy to be the person I am"; Gutierrez et al., 2002). Estimates of internal consistency and concurrent validity of the total RFL-YA and the five subscales are acceptable (see Gutierrez et al., 2000; Osman et al., 1999). Specifically, scores on the RFL-YA have been shown to be correlated with the Suicide Probability Scale (SPS; Cull \& Gill, 1992) and the Positive and Negative Affect Schedule (PANAS; Osman, Gutierrez, Kopper, Barrios, \& Chiros, 1998) in young adults (Gutierrez et al., 2002).

Modified Scale for Suicide Ideation (MSSI). The MSSI (Miller, Norman, Bishop, \& Dow, 1986) is an 18-item self-report measure that was constructed to assess the presence and severity of suicidal ideation within the past 2 weeks. Example items on the MSSI include "Have you been thinking of a way to kill yourself?" and "How strong or clear have your suicidal thoughts been?" Participants rate each item on a 4-point scale, with a higher score indicating a higher level of suicide ideation. Scores on the items are summed to obtain a total scale score. Higher scores on the MSSI have been found to be significantly correlated with greater hopelessness, depression, and measures of suicidal thoughts and behaviors (Pettit et al., 2009). The MSSI has shown good estimates of internal consistency, convergent validity, and discriminant validity in studies with clinical and nonclinical samples (Clum \& Yang, 1995; Wingate, Van Orden, Joiner, Williams, \& Rudd, 2005).

\footnotetext{
${ }^{1}$ We also conducted all analyses using the original BDI-II total score (i.e., including item 9) and observed an identical pattern of results.
} 
Table 1

Descriptives and Bivariate Associations Among Study Variables

\begin{tabular}{|c|c|c|c|c|c|c|c|c|c|c|}
\hline \multirow[b]{2}{*}{$\begin{array}{l}\text { Frequency/mean } \\
S D\end{array}$} & \multirow[b]{2}{*}{$\begin{array}{c}\text { Attempt } \\
2.1 \%\end{array}$} & \multirow[b]{2}{*}{$\begin{array}{c}\text { Ideation } \\
1.99 \\
(4.49)\end{array}$} & \multirow[b]{2}{*}{$\begin{array}{c}\text { Depression } \\
8.94 \\
(8.80)\end{array}$} & \multirow[b]{2}{*}{$\begin{array}{c}\text { Hopeless } \\
2.62 \\
(3.07)\end{array}$} & \multicolumn{6}{|c|}{$R F L-Y A$} \\
\hline & & & & & Total & $\begin{array}{c}\text { Family } \\
5.25 \\
(0.93)\end{array}$ & $\begin{array}{l}\text { Peer } \\
5.21 \\
(.93)\end{array}$ & $\begin{array}{c}\text { Cope } \\
5.34 \\
(0.81)\end{array}$ & $\begin{array}{c}\text { Future } \\
5.43 \\
(0.78)\end{array}$ & $\begin{array}{c}\text { Self } \\
5.19 \\
(0.96)\end{array}$ \\
\hline Attempt & - & & & & & & & & & \\
\hline Ideation & .50 & .91 & & & & & & & & \\
\hline Depression & .39 & .57 & .91 & & & & & & & \\
\hline Hopeless & .35 & .51 & .58 & .79 & & & & & & \\
\hline RFL-YA Total & -.37 & -.52 & -.49 & -.59 & .97 & & & & & \\
\hline RFL-YA Family & -.31 & -.41 & -.38 & -.47 & .84 & .94 & & & & \\
\hline RFL-YA Peer & -.30 & -.41 & -.38 & -.44 & .86 & .64 & .94 & & & \\
\hline RFL-YA Cope & -.38 & -.48 & -.42 & -.50 & .89 & .67 & .70 & .89 & & \\
\hline RFL-YA Future & -.36 & -.46 & -.43 & -.62 & .89 & .68 & .69 & .78 & .92 & \\
\hline RFL-YA Self & -.33 & .52 & -.51 & -.55 & .90 & .67 & .70 & .76 & .80 & .92 \\
\hline
\end{tabular}

Note. $S D=$ standard deviation; RFL = Reasons for Living for Young Adults; Family = RFL-YA Family Relations subscale; Peer = RFL-YA Peer Relations subscale; Cope = RFL-YA Coping Beliefs subscale; Future $=$ RFL-YA Future Expectations Subscale; Self = RFL-YA Positive Self-Evaluation subscale.

Pearson product moment correlations and point biserial correlations (one dichotomous and one continuous variable) are presented. Correlation coefficients all are significant at $p<.001$. Coefficient alphas are presented in bold. Past-year attempt is coded $0=$ absent, $1=$ present. Ideation $=$ total score (mean and standard deviation prior to transformation) of the Modified Scale for Suicide Ideation; Depression = total score of the Beck Depression Inventory-II (with ideation item removed); Hopeless = total score of the Beck Hopelessness Scale. $N=1075$.

Lifetime suicide attempt was assessed with one dichotomous (yes/no) question: "Have you ever tried to kill yourself or attempt suicide?" The question is a modification of the suicide attempt item used in the National Comorbidity Survey (Kessler, Borges, \& Walters, 1999), a nationally representative mental health survey. This item has served as an outcome in prior research (e.g., Conner, Houston, \& Swogger, 2009, Hills, Cox, McWilliams, \& Sareen, 2005, Vickers \& McNally, 2004) and shows high test-retest reliability (e.g., kappa $=.82$; Conner, Britton, Sworts, \& Joiner, 2007).

\section{Results}

\section{Data Screening}

Variable distributions were examined, revealing that the suicide ideation variable evidenced substantial positive skew and kurtosis ( skewness $=3.83$; kurtosis $=16.87$ ). These scores underwent a $\log$ transformation that resulted in acceptable levels of skew (1.38) and kurtosis (1.22; Kline, 2005). For ease of presentation and interpretation, the original mean and standard deviation of the suicidal ideation variable (i.e., prior to transformation) are presented in tables and text. However, all analyses utilized the transformed scores.

\section{Descriptives and Intercorrelations}

Demographic variables were not significantly related to the suicidal outcomes. Prior to conducting path analysis, bivariate correlations between the main variables of interest were examined (see Table 1). Given the large sample size and number of bivariate analyses, a Bonferroni correction for multiple tests (significance set at $p<0.001$ ) was applied. All variables were significantly correlated with one other and ranged from -.30 (peer relations with suicide attempt) to .80 (future expectations and positive self-evaluation RFL-YA). 


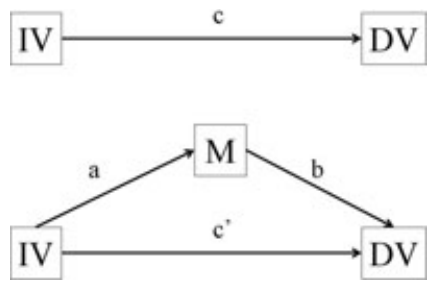

Figure 1. Total (c), Indirect (a X b), and Direct Effects ( $\left.\mathrm{c}^{\prime}\right)$ in Mediational Analysis. IV = independent variable; $\mathrm{M}=$ mediator; and $\mathrm{DV}=$ dependent variable.

\section{Definitions of Total, Indirect, and Direct Effects}

Mediation analysis, as described by Baron and Kenny (1986), Bollen (1989) and MacKinnon (2008), can be carried out within path analysis. As illustrated in Figure 1, the total effect (c) is defined as the effect of the independent variable on the outcome when paths involving the mediator are set to zero. The indirect effect $(a \mathrm{X} b)$ is defined as the effect of the independent variable on the outcome through the mediator and is also referred to as the mediated effect. The direct effect $\left(c^{\prime}\right)$ is the effect of the independent variable on the outcome when freely estimating the indirect effect. Mediated effects can be considered partial or full (Baron \& Kenny, 1986). Partial mediation is the case in which statistically significant indirect and direct effects are observed (i.e., the direct path is reduced in magnitude but is still greater than zero). Full mediation is the case in which a statistically significant indirect effect is observed, but the direct effect becomes nonsignificant (i.e., the direct effect equals zero).

\section{Analytic Procedure for Mediational Models}

Two series of mediational models (one model per dependent variable-suicide ideation and suicide attempt) were conducted in a path analytic framework using the Mplus Version 6.1 (B.O. Muthen \& L.K. Muthen, 1998-2011) software. Mplus can accommodate simultaneous modeling of multiple mediators and both continuous and dichotomous outcomes and provides estimates and significance tests of indirect effects using the Sobel test, which is appropriate for both continuous and dichotomous outcomes (Mackinnon, Lockwood, Brown, Wang, \& Hoffman, 2007; Mackinnon, Lockwood, Hoffman, West, \& Sheets, 2002; Muthen, 2011). For models with either dichotomous or continuous outcome, the same path analytic strategy was conducted, with different estimators. Specifically, for models including suicidal ideation as an outcome, model parameters were estimated by means of maximum likelihood estimation. For models including the dichotomous suicide attempt outcome variable, the models were fit to the data by means of MLR estimation, which provides maximum likelihood parameter estimates with standard errors and a chi-square test statistic that are robust to non-normality. The MLR standard errors are computed using a sandwich estimator. Sobel tests of indirect effects were conducted for both continuous and dichotomous outcomes.

For the first series of models, models were specified such that the mediator (RFL-YA total score) was regressed on correlated independent variables (depressive symptoms and hopelessness), while the suicidality outcome (ideation or attempt) was regressed on the mediator and the independent variables simultaneously. The second series of models was identical to the first with the exception that the RFL-YA total score was replaced by the five RFL-YA subscales, serving as simultaneous mediators. Correlations between disturbances of the RFL-YA subscales were specified. Model fit indices are not presented given the just-identified nature of each of the models. Of note, within each of these series, an estimate of total effects (i.e., the suicide outcome regressed on depressive symptoms and hopelessness) was obtained by conducting an identical set of models with the exception that all parameters (e.g., regression paths and covariances) involving the mediator(s) were set to zero. 


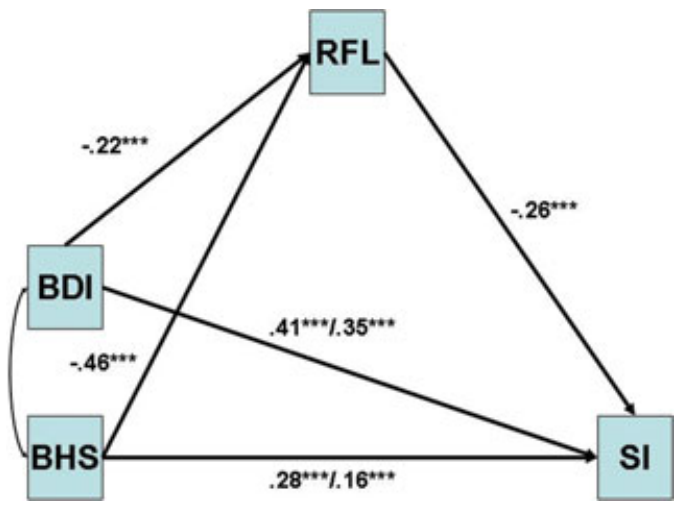

Figure 2. RFL-YA Total Score as a Mediator of Depressive Symptoms and Hopelessness associations with Suicidal Ideation. BDI = Beck Depression Inventory-II; BHS = Beck Hopelessness Scale; SI = Modified Scale for Suicide Ideation; Numbers represent standardized betas. The first number prior to the slash represents the $\mathrm{c}$ path (i.e., when paths involving mediators are fixed at zero). ${ }^{*} \mathrm{p}<.05 .{ }^{* *} \mathrm{p}<.01 .{ }^{* * *} \mathrm{p}<$ .001 .

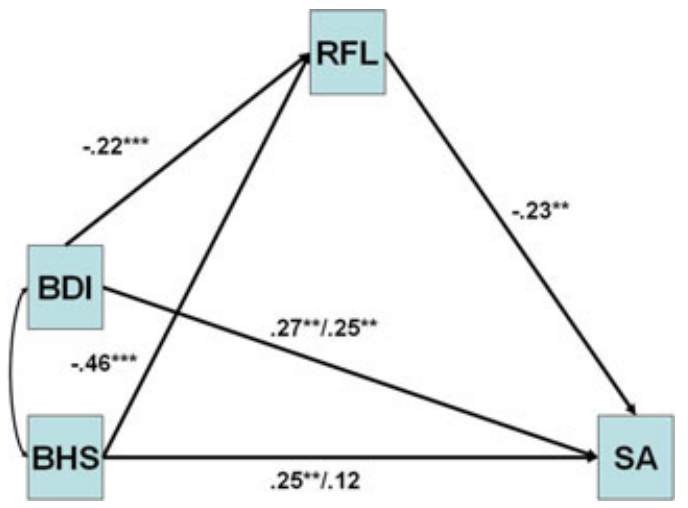

Figure 3. RFL-YA Total Score as a Mediator of Depressive Symptoms and Hopelessness associations with Suicide Attempt. BDI = Beck Depression Inventory-II; BHS = Beck Hopelessness Scale; SA = Suicide Attempt within the past 12 months. Standardized coefficients are presented. Coefficients of paths to suicide attempt are standardized logits. The first number prior to the slash represents the $\mathrm{c}$ path (i.e., when paths involving mediators are fixed at zero). ${ }^{*} \mathrm{p}<.05 .{ }^{* *} \mathrm{p}<.01 .{ }^{* * *} \mathrm{p}<.001$.

\section{Meditational Models Including RFL-YA Total Scores}

For the first series of path analytical models, results indicate that relations between both independent variables with current suicidal ideation (see Figure 2) were partially mediated by RFL-YA total scores (indirect effects: hopelessness $\beta=.12, p<.001$; depressive symptoms $\beta=.06, p<.001$ ). Next, when examining suicidal behavior (see Figure 3), results indicate that the RFL-YA fully mediates the relation between hopelessness and past-year suicide attempt history (indirect effect: $\beta=.11, p<.05$ ) and partially mediates the depressive symptoms-suicide attempt relation (indirect effect: depression $\beta=.05, p<.05$ ). Across these models, the percent of variance accounted for $\left(R^{2}\right)$ was .38 for RFL-YA total score, .42 for suicidal ideation, and .25 for suicide attempt.

\section{Meditational Models Including RFL-YA Subscales}

For the second series of path analytical models, the RFL-YA total scores were replaced by its five subscales to serve as simultaneous mediators. For each model, the disturbances of the endogenous RFL-YA variables were correlated. When modeling current suicidal ideation 


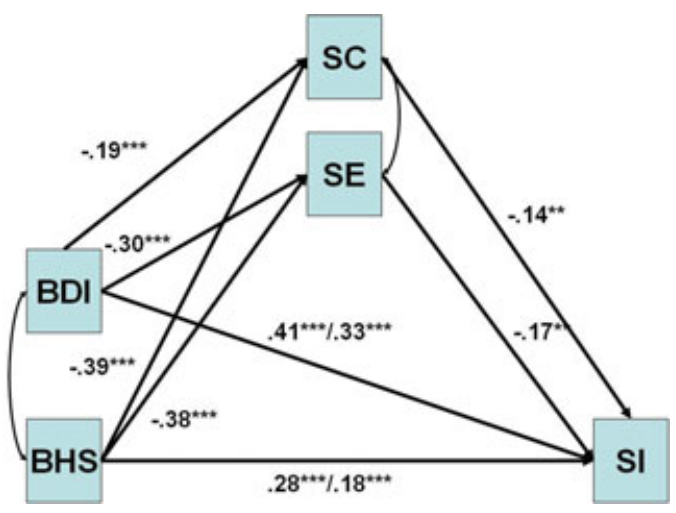

Figure 4. RFL-YA Subscales as Mediators of Depressive Symptoms and Hopelessness associations with Suicidal Ideation. BDI = Beck Depression Inventory-II; BHS = Beck Hopelessness Scale; SI = Modified Scale for Suicide Ideation; SC = Survival and Coping Beliefs; SE = Positive Self-Evaluation. Numbers represent standardized betas. The first number prior to the slash represents the $\mathrm{c}$ path (i.e., when paths involving mediators are fixed at zero). Paths of other RFL-YA subscales are not displayed (since all of these $\mathrm{b}$ paths were $\mathrm{ns})$ but were included in the model. ${ }^{*} \mathrm{p}<.05 .{ }^{* *} \mathrm{p}<.01 . * * * \mathrm{p}<.001$.

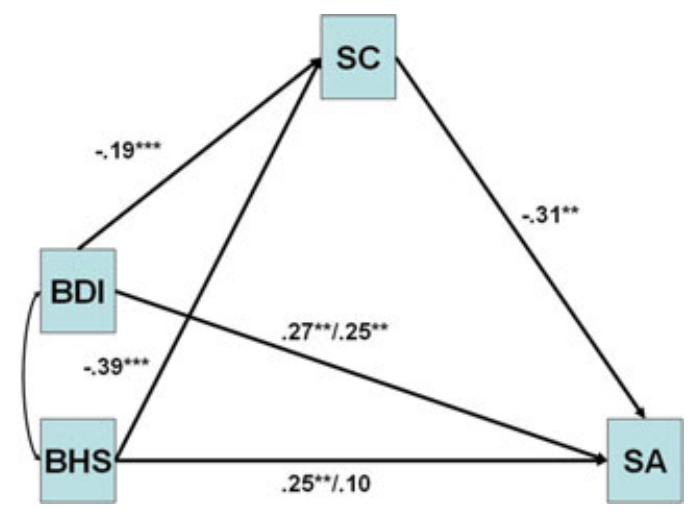

Figure 5. RFL-YA Subscales as Mediators of Depressive Symptoms and Hopelessness associations with Suicide Attempt. BDI = Beck Depression Inventory-II; BHS = Beck Hopelessness Scale; SA = Suicide Attempt within the past 12 months; SC = Survival and Coping Beliefs. Standardized coefficients are presented. Coefficients of paths to suicide attempt are standardized logits. The first number prior to the slash represents the c path (i.e., when paths involving mediators are fixed at zero). Paths of other RFL-YA subscales are not displayed (since all of these $b$ paths were $n s$ ) but were included in the model. ${ }^{*} \mathrm{p}<.05$. $* * \mathrm{p}<.01 . * * * \mathrm{p}<.001$

(see Figure 4), results indicate that the RFL-YA subscales, which contributed to the partially mediated effects involving the RFL-YA total score, include SCB (indirect effects: hopelessness, $\beta$ $=.06, p<.01$; depressive symptom, $\beta=.03 p<.05$ ) and positive self-evaluation (indirect effects: hopelessness, $\beta=.07, \mathrm{p}<.01$; depressive symptoms, $\beta=.05 \mathrm{p}<.01$ ). When modeling past-year suicide attempt history (see Figure 5), SCB was the only RFL-YA subscale that contributed to mediated effects involving the RFL-YA total score. Specifically, SCB is a full mediator of the hopelessness and suicide attempt relation (indirect effect: $\beta=.12, p<.001$ ), as well as a partial mediator between the depressive symptoms and suicide attempt relation (indirect effect: $\beta=$ $.06, p<.01)$. Across these models, the percent of variance accounted for $\left(R^{2}\right)$ by SCB was .28 , positive self-evaluation was .36, suicidal ideation was .42 , and suicide attempt was $.26 .^{2}$

\footnotetext{
${ }^{2}$ As a test of gender differences of regression paths within these path models, we also conducted multigroup analyses in Mplus. For each model (see Figures 2 to 5), the model was estimated separately for men and
} 


\section{Discussion}

The goals of this study were to determine (a) whether total RFL simultaneously mediates the relation between risk factors (hopelessness and depressive symptoms) and both suicidal ideation and attempt, and (b) whether specific categories of RFL uniquely mediate the relation between risk factors and suicide ideation and attempt. This study is the first, to our knowledge, to examine specific categories of RFL as simultaneous mediators of the relations between key risk factors (depressive symptoms and hopelessness) and suicidal thoughts and suicide attempts.

First, consistent with previous research, the current study found that depressive symptoms and hopelessness were positively associated with suicidal thoughts and behavior (Haney et al., 2012; Lamis \& Lester, 2012) and negatively associated with RFL (Dean \& Range, 1999; Dyck, 1991; Ellis \& Lamis, 2007; Gutierrez et al., 2002; Malone et al., 2000; Rich \& Bonner, 1987). In turn, RFL were negatively associated with suicidal thoughts and behavior (Chapman et al., 2005; Dean \& Range, 1999; Gutierrez et al., 2002; Dyck, 1991; Linehan et al., 1983; Mann et al., 1999; Malone et al., 2000; Rich \& Bonner, 1987). Second, the results of the present study strongly support our hypotheses that RFL would partially account for the relations between risk factors and suicidal ideation and attempt, suggesting that other influences also account for these relations. However, there was one notable exception to our hypothesized results: RFL fully accounted for the relation between hopelessness and suicide attempt. As such, our findings highlight the particular relevance of the RFL mechanism in explaining the link between hopelessness and suicidal actions versus suicidal ideation. However, it is important to underscore that these findings are preliminary and need replication.

Next, we examined which RFL subscale contributed to the total RFL mediated effects. Consistent with our hypotheses, we found that SCB RFL, belief in the importance of dealing with and finding solutions for adversity, uniquely contributed to the total RFL mediated effects, and thus at least partially accounted for relations between risk factors and both suicide ideation and attempt. Research shows that low reliance on strategies that address the direct management of the problem is associated with suicidal ideation (Elliott \& Frude, 2001; Schotte \& Clum, 1987) and suicidal behavior (Lauer, de Man, Marquez, \& Ades, 2008), and a body of research shows that interventions designed to increase problem-solving and coping skills demonstrated a reduction in suicidal ideation (Pollock \& Williams, 1998) and suicidal behavior (Joiner, Voelz, \& Rudd, 2001; Ghahramanlou-Holloway, Bhar, Brown, Olsen, \& Beck, 2012) although this is not invariably so (Fremouw, Callahan, \& Kashden, 1993; Kashden, Fremouw, Callahan, \& Franzen, 1993).

Of note, SCB was the only RFL factor that contributed to every RFL total mediated effect, and SCB was the only RFL subscale to fully mediate the relation between hopelessness and suicide attempt. One potential reason why the inclusion of SCB reduced the hopelessnesssuicide attempt association to nonsignificance, is that SCB RFL are suicide-specific protective beliefs that tap important content beyond hopelessness (Strosahl et al., 1992). Thus, research focusing on the feasibility of increasing endorsement of the importance of coping with and finding solutions for problems as a reason to not kill oneself is a promising area to consider when enhancing prevention efforts aimed at reducing suicidal thoughts and behaviors.

However, surprisingly, self-evaluative RFL was an additional RFL subscale that partially accounted for associations between risk factors and suicidal ideation. Our finding that having decreased reasons for living, concerning feeling good and having respect for oneself, was associated with increased levels of risk factors and suicidal thoughts is consistent with research finding that having a negative self-view (Beck, 1967) or a self-discrepancy between one's ideal and actual self (Higgins, 1987), increased risk for suicidal thoughts and behaviors (Beck \& Weishaar, 1990;

women without constraining paths to equality across groups (an unconstrained baseline model). The chisquare for the unconstrained model was then compared with the chi-square from a model in which all paths were constrained to be equal for men and women (constrained model). There was not a significant difference in chi-squares between constrained and unconstrained models displayed in Figure $2, \chi^{2}(5)=1.62, p=.89$, Figure $3, \chi^{2}(5)=3.76, p=.58$, Figure $4, \chi^{2}(17)=15.91, p=.53$, and Figure $5, \chi^{2}(17)=17.83, p=.40$, respectively. 
Cornette, Strauman, Abramson, \& Busch 2009). Interestingly, our preliminary work demonstrated that self-evaluative beliefs contributed only to RFL total score mediated effects involving suicidal ideation (and not suicide attempts). Future research is needed to further understand key boundaries of self-evaluative RFL as a potential important explanatory factor of associations between traditional risk factors and suicidal outcomes.

Taken together, our findings have important implications for both suicide research and clinical work with suicidal patients. First, in regard to future research, much of the extant literature focuses on negative suicide risk factors, while there has been a relatively limited focus on the presence of protective factors for suicide. Determining the extent to which protective factors are present is an important complement to obtaining information about more traditional negative risk factors in assessing risk potential for suicidal thoughts or engaging in suicidal behaviors. It is important to highlight that we are not denying the extreme value and utility of the constructs of depression and hopelessness as risk factors for suicidal thoughts and behavior. Instead, we are suggesting that RFL is another important cognitive factor and that this construct appears to account for at least some of the association between known suicide risk factors and suicidality.

Specifically, our findings demonstrate that (a) lower RFL are related to higher levels of risk factors (depressive symptoms and hopelessness) and higher levels of suicidal outcomes and (b) lower RFL mediate the risk factor-suicidality relation (such that higher levels of risk factors are then more weakly associated with higher levels of suicidal outcomes). Consequently, we believe that RFL deserves increased attention as a critical component in comprehensive theoretical models of suicidality. Future researchers should consider employing longitudinal designs to examine the possible underlying mechanisms in the relation between established risk factors (e.g., depression, hopelessness) and suicidality.

Second, in terms of the clinical implications of our results, findings that depressive symptoms and hopelessness continue to be distinguishing risk factors for suicidal ideation and attempts highlights the importance of assessing and preventing future recurrences of mood disorders. Routine screenings of depressive and hopelessness symptoms could take place during doctor's visits (regardless of presenting complaint), college orientations, and community screening days, and this research reinforces the importance of adequate follow-up, referral, and monitoring of depressive and hopeless symptoms to prevent future suicidal behavior.

Another factor, RFL, is also important to consider when treating young adults at high-risk for suicidal thoughts and behaviors. RFL may be useful in the development of a treatment plan, whereby a patient's individual strengths and adaptive RFL against suicide would be identified. Further, RFL is a potentially modifiable factor (Brown, Bryan, \& Holloway, 2012) and interventions that focus on bolstering/generating RFL, such as a novel brief cognitive therapy (Brown et al., 2012), could prove fruitful in increasing RFL and decreasing suicidal thoughts and future suicide attempts among high-risk groups. Identification of more refined treatment targets of suicidality among depressed or hopeless individuals is intriguing and directly targeting an increase in this protective factor may help to weaken the link between depression, hopelessness, and suicidal thoughts and behavior. Clinicians and mental health professionals may need to focus their attention on modifying RFL, in addition to reducing depression and hopelessness, when treating suicidal patients; however, this remains an empirical question.

Further, a growing body of research on the paradoxical consequences of efforts to change, suppress, or control internal experiences (including thoughts and emotions; Hayes, Strosahl, $\&$ Wilson, 1999) has led to the development of acceptance-based behavioral therapies focused on increasing contact with things that are meaningful to that individual, rather than directly changing thoughts or emotions per se (Hayes et al., 1999; Linehan, 1993). A focus on increasing or strengthening one's connection with RFL is consistent with this approach and may be a fruitful complement to existing treatments for suicidality.

Although the current study adds to the growing literature examining factors that may account for well-established suicide risk factors and suicidal thoughts and behavior, there are limitations to this study that deserve comment and suggest areas for future research. First, our sample included a large sample of college students and it is unknown how these results would generalize to young adults who are not students, as well as to young people with documented psychiatric histories. Specifically, we had a relatively healthy college student sample and the average ideation, 
depression, and hopelessness scores were below clinical cutoffs, while the average RFL-YA scores were higher than clinical cutoffs.

Further, the current study had a low base rate of past-year suicide attempt (although this rate is consistent with prior literature focusing on college students; Barrios et al., 2000). Thus, within a college student population, risk and protective factors for suicidal thoughts and attempts may not be as easily identifiable, as they might be for clinical populations, which may limit the generalizability of the present findings to other populations. Replication of our findings across populations would increase our confidence that RFL at least partially mediates the relation between traditional risk factors and suicidal ideation and behavior. Second, similar to prior investigations with a college student population (Freedenthal, Lamis, Osman, Kahlo, \& Gutierrez, 2011; Lamis \& Malone, 2011), the current study was limited by including only self-report data collected online.

Although the current study had excellent estimates of internal consistency, and associations between study variables were within the expected direction, we did not include multiple modes of assessment (e.g., interviews). In addition, we included only a single item to measure a past-year suicide attempt, instead of a multiple item assessment. Future studies should consider using multiple items to assess suicidal behavior and a multimodal data collection strategy with college students, such as conducting face-to-face interviews, in addition to administering self-report questionnaires to examine for the potential of method effects.

Third, given our cross-sectional design, the temporal ordering of constructs cannot be determined. It remains possible that RFL influences level of depressive symptoms and hopelessness, and not vice versa. Prospective event-based assessments are needed to clarify the role of depression, hopelessness, RFL, and suicidal ideation and attempts. Finally, validated self-report measures of psychopathology were used. Future studies should replicate these findings with validated structured interviews to assess diagnostic features within hypothesized relations.

Despite these limitations, the current study maintains important empirical and clinical implications. Empirically, our study is one of the initial examinations of RFL (and RFL subscales) as mediators of relations between well-established risk factors and suicidality among young adults. This study also provides suggestive evidence regarding the ways in which these factors may operate to produce suicide-related outcomes. In terms of clinical implications, a greater understanding of the role of RFL in suicidal behavior can help to develop more targeted individualized interventions for high-risk suicidal individuals.

\section{References}

American College Health Association. (2011). ACHA-National College Health Assessment II: Reference group executive summary Spring 2011. Hanover, MD: Author.

Baron, R. M., \& Kenny, D. A. (1986). The moderator-mediator variable distinction in social psychological research: Conceptual, strategic, and statistical considerations. Journal of Personality and Social Psychology, 51, 1173-1182.

Barrios, L. C., Everett, S. A., Simon, T. R., \& Brener, N. D. (2000). Suicide ideation among US college students: Associations with other injury risk behaviors. Journal of American College Health, 48, 229-233.

Beck, A. T. (1967). Depression: Clinical, experimental, and theoretical aspects. New York: Harper \& Row.

Beck, A. T., Steer, R. A., \& Brown, G. K. (1996). Manual for the Beck Depression Inventory: Second edition. San Antonio, TX: The Psychological Corporation.

Beck, A. T., \& Weishaar, M. E. (1990). Suicide risk assessment and prediction. Crisis: The Journal Of Crisis Intervention And Suicide Prevention, 11, 22-30.

Beck, A., Weissman, A., Lester, D., \& Trexler, L. (1974). The measurement of pessimism: The Hopelessness Scale. Journal of Consulting and Clinical Psychology, 42, 861-865.

Bisconer, S. W. \& Gross, D. M. (2007). Assessment of suicide risk in a psychiatric hospital. Professional Psychology: Research and Practice, 38, 143-149.

Bollen, K. A. (1987). Structural equation models with latent variables. New York: John Wiley.

Brown, G., Bryan, C. J., \& Holloway, M. (2012). Cognitive behavior therapy for reducing suicide risk with veterans and military service members. Panel presented at the annual meeting of the American Association of Suicidology, Baltimore, MD. 
Brown, G. K., Henriques, G. R., Sosdjan, D., \& Beck, A. T. (2004). Suicide intent and accurate expectations of lethality: Predictors of medical lethality of suicide attempts. Journal of Consulting and Clinical Psychology, 72, 1170-1174.

Centers for Disease Control and Prevention. (2010). Injury prevention \& control: Data \& Statistics (WISQARS). Retrieved from http://www.cdc.gov/injury/wisqars/fatal.html

Chapman, A. L., Specht, M. W., \& Cellucci, T. (2005). Factors associated with suicide attempts in female inmates: The hegemony of hopelessness. Suicide and Life-Threatening Behavior, 35, 558-569.

Clum, G. A., \& Yang, B. (1995). Additional support for the reliability and validity of the Modified Scale for Suicide Ideation. Psychological Assessment, 7, 122-125.

Conner, K. R., Britton, P. C., Sworts, L., \& Joiner, T. (2007). Suicide attempts among individuals with opiate dependence: The role of belonging. Addictive Behaviors, 32, 1395-1404.

Conner, K. R., Houston, R. J., \& Swogger, M. A. (2009). A test of the reactive aggression-suicidal behavior hypothesis: Is there a case for proactive aggression? Journal of Abnormal Psychology, 118, 235-240.

Cornette, M. M., Strauman, T. J., Abramson, L. Y., \& Busch, A. M. (2009). Self-discrepancy and suicidal ideation. Cognition and Emotion, 23, 504-527.

Cull, J. G., \& Gill, W. S. (1982). Suicide Probability Scale. Los Angeles, CA: Western Psychological Services.

Dean, P., \& Range, L. M. (1999). Testing the Escape Theory of Suicide in an outpatient clinical population. Cognitive Therapy and Research, 23, 561-572.

Dogra, A. K., Basu, S., \& Das, S. (2011). Impact of the meaning in life and reasons for living to hope and suicidal ideation: A study among college students. Journal of Projective Psychology and Mental Health, $18,89-102$.

Drum, D. J., Brownson, C., Burton Denmark, A., \& Smith, S. E. (2009). New data on the nature of suicidal crises in college students: Shifting the paradigm. Professional Psychology: Research and Practice, 40, 213-222.

Drum, D. J., \& Burton Denmark, A. (2011). College suicide prevention programs and interventions. In D. A. Lamis \& D. Lester (Eds.), Understanding and preventing college student suicide (pp. 255-272). Charles C. Thomas Publishers: Springfield, IL.

Dyck, M. J. (1991). Positive and negative attitudes mediating suicide ideation. Suicide and Life-Threatening Behavior, 21, 360-373.

Elliott, J. L., \& Frude, N. (2001). Stress, coping styles, and hopelessness in self-poisoners. Crisis: The Journal of Crisis Intervention and Suicide Prevention, 22, 20-26.

Ellis, J. B., \& Lamis, D. A. (2007). Adaptive characteristics and suicidal behavior: A gender comparison of young adults. Death Studies, 31, 845-854.

Freedenthal, S., Lamis, D. A., Osman, A., Kahlo, D., \& Gutierrez, P. M. (2011). Evaluation of the psychometric properties of the Interpersonal Needs Questionnaire-12 in samples of men and women. Journal of Clinical Psychology, 67, 609-623.

Fremouw, W., Callahan, T., \& Kashden, J. (1993). Adolescent suicidal risk: Psychological, problem solving, and environmental factors. Suicide and Life-Threatening Behavior, 23, 46-54.

Galfalvy, H., Oquendo, M. A., Carballo, J. J., Sher, L., Grunebaum, M. F., Burke, A., \& Mann, J. (2006). Clinical predictors of suicidal acts after major depression in bipolar disorder: A prospective study. Bipolar Disorders, 8, 586-595.

Ghahramanlou-Holloway, M. M., Bhar, S. S., Brown, G. K., Olsen, C. C., \& Beck, A. T. (2012). Changes in problem-solving appraisal after cognitive therapy for the prevention of suicide. Psychological Medicine, 42, 1185-1193.

Gutierrez, P., Osman, A., Barrios, F., Kopper, B., Baker, M., \& Haraburda, C. (2002). Development of the Reasons for Living Inventory for Young Adults. Journal of Clinical Psychology, 58, 339-357.

Gutierrez, P. M., Osman, A., Kopper, B. A., Barrios, F. X., \& Bagge, C. L. (2000). Suicide risk assessment in a college student population. Journal of Counseling Psychology, 47, 403-413.

Haney, E. M., O’Neil, M. E., Carson, S., Low, A., Peterson, K., Denneson, L. M., \& .. K Kansagora, D. (2012). Suicide risk factors and risk assessment tools: A systematic review. VA-ESP Project \#05-225.

Hayes, S. C., Strosahl, K., \& Wilson, K. G. (1999). Acceptance and Commitment Therapy: An experiential approach to behavior change. New York, NY: The Guilford Press.

Hills, A. L., Cox, B. J., McWilliams, L. A., \& Sareen, J. (2005). Suicide attempts and externalizing psychopathology in a nationally representative sample. Comprehensive Psychiatry, 46, 334-339. 
Jobes, D. A. (2006). Managing suicidal risk: A collaborative approach. New York, NY: Guilford Press.

Joiner, T. E. (2005). Why people die by suicide. Cambridge, MA: Harvard University Press.

Joiner, T. R., Voelz, Z. R., \& Rudd, M. (2001). For suicidal young adults with comorbid depressive and anxiety disorders, problem-solving treatment may be better than treatment as usual. Professional Psychology: Research and Practice, 32, 278-282.

Kashden, J., Fremouw, W. J., Callahan, T. S., \& Franzen, M. D. (1993). Impulsivity in suicidal and nonsuicidal adolescents. Journal of Abnormal Child Psychology, 21, 339-353.

Keilp, J. G., Grunebaum, M. F., Gorlyn, M., LeBlanc, S., Burke, A. K., Galfalvy, H., \& . . Mann, J. (2012). Suicidal ideation and the subjective aspects of depression. Journal of Affective Disorders, 140, 75-81.

Kessler, R. C., Borges, G., \& Walters, E. E. (1999). Prevalence of and risk factors for lifetime suicide attempts in the National Comorbidity Survey. Archives of General Psychiatry, 56, 617-626.

Kline, R. B. (2005). Principles and practice of structural equation modeling (2nd ed.). New York, NY: Guilford Press.

Lamis, D. A., \& Lester, D. (2012). Risk factors for suicidal ideation among African American and European American college women. Psychology of Women Quarterly, 36, 337-349.

Lamis, D. A., \& Malone, P. S. (2011). Alcohol-related problems and risk of suicide among college students: The mediating roles of belongingness and burdensomeness. Suicide and Life-Threatening Behavior, 41, 543-553.

Lauer, S., de Man, A. F., Marquez, S., \& Ades, J. (2008). External locus of control, problem-focused coping and attempted suicide. North American Journal of Psychology, 10, 625-632.

Linehan, M. M. (1993). Cognitive-behavioral treatment of borderline personality disorder. New York, NY: Guilford Press.

Linehan, M. M., Goodstein, J. L., Nielsen, S. L., \& Chiles, J. A. (1983). Reasons for staying alive when you are thinking of killing yourself: The Reasons for Living Inventory. Journal of Consulting and Clinical Psychology, 51, 276-286.

MacKinnon, D. P. (2008). An introduction to statistical mediation analysis. New York, NY: Lawrence Erlbaum Associates.

MacKinnon, D. P., Lockwood, C. A., Brown, C. H., Wang, W., \& Hoffman, J. M. (2007). The intermediate endpoint effect in logistic and probit regression. Clinical Trials, 4, 499-513.

MacKinnon, D. P., Lockwood, C. M., Hoffman, J. M., West, S. G., \& Sheets, V. (2002). A comparison of methods to test mediation and other intervening variable effects. Psychological Methods, 7, 83-104.

Malone, K. M., Oquendo, M. A., Haas, G. L., Ellis, S. P., Li, S., \& Mann, J. J. (2000). Protective factors against suicidal acts in major depression: Reasons for living. The American Journal of Psychiatry, 157, 1084-1088.

Mann, J., Waternaux, C., Haas, G. L., \& Malone, K. M. (1999). Toward a clinical model of suicidal behavior in psychiatric patients. The American Journal of Psychiatry, 156, 181-189.

Miller, I. W., Norman, W. H., Bishop, S. B., \& Dow, M. G. (1986). The Modified Scale for Suicide Ideation: Reliability and validity. Journal of Consulting and Clinical Psychology, 54, 724-725.

Muthén, L. K., \& Muthén, B. O. (1998-2011). Mplus 6.1 [Computer software]. Los Angeles, CA: Author.

Muthén, B. O. (2011). Applications of causally defined direct and indirect effects in mediation analysis using SEM in Mplus. Manuscript submitted for publication.

Naragon-Gainey, K., Watson, D., \& Markon, K. E. (2009). Differential relations of depression and social anxiety symptoms to the facets of extraversion/positive emotionality. Journal of Abnormal Psychology, 118, 299-310.

National Institute of Mental Health. (2011). Suicide in the U.S.: Statistics and Prevention. Retrieved from http://www.nimh.nih.gov/health/publications/suicide-in-the-us-statistics-and-prevention/index.shtml

Osman, A., Gutierrez, P., Kopper, B., Barrios, F., \& Chiros, C. (1998). The Positive and Negative Suicide Ideation Inventory: Development and validation. Psychological Reports, 82, 783-793.

Osman, A., Kopper, B. A., Barrios, F. X., Gutierrez, P. M., \& Bagge, C. L. (2004). Reliability and validity of the Beck Depression Inventory-II with adolescent psychiatric inpatients. Psychological Assessment, $16,120-132$.

Osman, A., Kopper, B. A., Linehan, M. M., Barrios, F. X., Gutierrez, P. M., \& Bagge, C. L. (1999). Validation of the Adult Suicidal Ideation Questionnaire and the Reasons for Living Inventory in an adult psychiatric inpatient sample. Psychological Assessment, 11, 115-123. 
Pettit, J., Garza, M., Grover, K., Schatte, D., Morgan, S., Harper, A., \& Saunders, A. E. (2009). Factor structure and psychometric properties of the Modified Scale for Suicidal Ideation among suicidal youth. Depression and Anxiety, 26, 769-774.

Pollock, L. R., \& Williams, M. G. (1998). Problem solving and suicidal behavior. Suicide and LifeThreatening Behavior, 28, 375-387.

Pompili, M., Girardi, P., Tatarelli, G., \& Taterelli, R. (2006). Suicidal intent in single-car accident drivers: Review and new preliminary findings. Crisis: The Journal of Crisis Intervention and Suicide Prevention, 27, 92-99.

Pukay-Martin, N. D., Pontoski, K. E., Maxwell, M. A., Calhoun, P. S., Dutton, C. E., Clancy, C. P. ... Beckman, J. C. (2012). The influence of depressive symptoms on suicidal ideation among U.S. VietnamEra and Afghanistan/Iraq-Era veterans with posttraumatic stress disorder. Journal of Traumatic Stress, 25, 578-582.

Rich, A. R., \& Bonner, R. L. (1987). Concurrent validity of a stress-vulnerability model of suicidal ideation and behavior: A follow-up study. Suicide and Life-Threatening Behavior, 17, 265-270.

Schotte, D. E., \& Clum, G. A. (1987). Problem-solving skills in suicidal psychiatric patients. Journal of Consulting and Clinical Psychology, 55, 49-54.

Schotte, D. E., Cools, J., \& Payvar, S. (1990). Problem-solving deficits in suicidal patients: Trait vulnerability or state phenomenon? Journal of Consulting and Clinical Psychology, 58, 562-564.

Steed, L. (2001). Further validity and reliability evidence for Beck Hopelessness Scale scores in a nonclinical sample. Educational and Psychological Measurement, 61, 303-316.

Strosahl, K., Chiles, J. A., \& Linehan, M. (1992). Prediction of suicide intent in hospitalized parasuicides: Reasons for living, hopelessness, and depression. Comprehensive Psychiatry, 33, 366-373.

Vickers, K., \& McNally, R. J. (2004). Panic disorder and suicide attempt in the National Comorbidity Survey. Journal of Abnormal Psychology, 113, 582-591.

Wang, M. C., Lightsey, O. R., Pietruszka, T., Uruk, A. C., \& Wells, A. G. (2007). Purpose in life and reasons for living as mediators of the relationship between stress, coping, and suicidal behavior. The Journal of Positive Psychology, 3, 195-204.

Wenzel, A., Brown, G. K., \& Beck, A. T. (2008). Cognitive therapy for suicidal patients: Scientific and clinical applications. Washington, DC: APA Books.

Williams, J. M., Barnhofer, T., Crane, C., \& Beck, A. T. (2005). Problem solving deteriorates following mood challenge in formerly depressed patients with a history of suicidal ideation. Journal of Abnormal Psychology, 114, 421-431.

Wingate, L. R., Van Orden, K. A., Joiner, T. E., Williams, F. M., \& Rudd, M. D. (2005). Comparison of compensation and capitalization models when treating suicidality in young adults. Journal of Consulting and Clinical Psychology, 73, 756-762.

Xu, J., Kochanek, K. D., Murphy, S. L., \& Tejada-Vera, B. (2010). Deaths: Final Data for 2007. National Vital Statistics Reports, 58. Retrieved from http://www.cdc.gov/nchs/data/nvsr/nvsr58/nvsr58_19.pdf 